\title{
Zuwanderung in Kommunen - Steuerung durch Integrationskonzepte
}

Dieter Filsinger \& Maximilian Filsinger

\begin{abstract}
Deutschland hat in letzten Jahren einen starken Anstieg an Zuwanderung verzeichnet, die sowohl Fluchtmigration als auch Migration im Rahmen der Europäischen Union beinhaltet. Gleichwohl steht die Bundesrepublik Deutschland vor demografischen Herausforderungen, zu deren Lösung Zuwanderung einen Beitrag leisten soll. Die Stadtpolitik steht in besonderem Fokus, muss sie doch vor Ort die Auswirkungen der Zuwanderung und des demografischen Wandels angehen, ohne die großen Linien der Politik maßgeblich mitbestimmen zu können. In diesem Beitrag gehen wir der Frage nach, ob in deutschen kreisfreien Städten und Landkreisen Zuwanderungskonzepte zu finden sind, die sich einer Förderung von Zuwanderung im Sinne einer Anwerbung widmen. Unsere empirische Untersuchung zeigt, dass explizite Zuwanderungskonzepte kaum vorhanden sind. Vielmehr werden strategische Integrationskonzepte implementiert, die die Integration der Zugewanderten fördern und systematisieren sollen.
\end{abstract}




\section{Einleitung}

Die Bundesrepublik Deutschland hatte in den letzten Jahren - wie bereits auch in den 1990er Jahren - einen starken Anstieg der Zuwanderung zu verzeichnen (Bundesamt für Migration und Flüchtlinge 2017), wobei vor allem an die Fluchtzuwanderung, aber auch an die Arbeitsmigration aus der Europäischen Union (EU) zu denken ist. Diese Zuwanderungsbewegungen sind im Zusammenhang mit der demografischen Herausforderung zu sehen, die darin besteht, dass niedrige Geburtenraten zu einem Bevölkerungsrückgang führen (Bundesregierung 2015a). Erwartet wird ein erheblicher Fachkräftemangel. Viele deutsche Städte und Regionen stehen deshalb vor ökonomischen und sozialen Herausforderungen (Bundesinstitut für Bau-, Stadt-, und Raumforschung (BBSR) 2017a; 2017b). Ein Beitrag zur Lösung wird in Politik, Wirtschaft und Wissenschaft in weiterer Zuwanderung gesehen, die allerdings durch entsprechende Strategien und Konzepte gesteuert werden soll (Sachverständigenrat deutscher Stiftungen für Integration und Migration (SVR) 2018). Gleichzeitig ist jedoch in der Politik umstritten, wieviel und welche Zuwanderung in Betracht zu ziehen ist.

Vor diesem Hintergrund untersuchen wir empirisch, ob in deutschen kreisfreien Städten und Landkreisen Zuwanderungskonzepte aufzufinden sind, die sich einer Förderung von Zuwanderung im Sinne einer Anwerbung widmen. Basierend auf einer breit angelegten und systematischen Internetrecherche finden wir jedoch kaum Hinweise auf explizite kommunale Zuwanderungskonzepte. Mögliche Gründe sehen wir in der Widersprüchlichkeit der Bundespolitik Deutschland, die zwischen Willkommens- und Abschottungspolitik changiert und somit Bedingungen schafft, die für eine qualifizierte Zuwanderung nicht förderlich sind. Darüber hinaus steht die Bearbeitung der Folgen der Fluchtmigration der Jahre 2015 und 2016, ebenso die der Arbeitsmigration aus der EU im Vordergrund. Zudem sind 
liegengebliebene Aufgaben früherer Zu- bzw. Einwanderung zu bearbeiten (Filsinger 2018a; Filsinger/Gesemann 2017).

Auf Basis einer weiteren Recherche zu Integrationskonzepten konnten wir feststellen, dass Kommunen verstärkt im Feld der Integration strategische Konzepte beschließen. Kreisfreie Städte und Landkreise versuchen mit Hilfe von solchen strategischen Integrationskonzepten die sich dauerhaft stellenden Integrationsaufgaben zu lösen und gleichzeitig Steuerungspotentiale für eine erwartbare, zukünftige Zuwanderung zu gewinnen. Vor diesem Hintergrund kommen wir zu der Einschätzung, dass die kommunale integrationspolitische Governance als wichtiger Faktor im Zusammenhang mit zukünftigen

Zuwanderungsbewegungen betrachtet werden muss.

\section{Migration und Integration als kommunale Politikfelder}

Zur Analyse von Migrationsprozessen sind Unterscheidungen, so etwa Zuwanderung, Durchwanderung, Einwanderung, Abwanderung, Rückwanderung, Auswanderung, vorzunehmen (Pries 2013). In unserem Zusammenhang ist insbesondere die Unterscheidung von Zuwanderung- und Einwanderung von Interesse. ,Zuwanderung' meint allgemein den Prozess der Ankunft von Personen aus anderen Staaten, wobei offenbleibt, inwieweit die Zugewanderten in der Aufnahmegesellschaft längerfristig bleiben wollen bzw. können. Von Einwanderung ist zu sprechen, wenn Zugewanderte eine dauerhafte Perspektive in der Einwanderungsgesellschaft erkennen (können), wobei Pries (2008: 188) darauf hinweist, dass Migration als „ein zukunfts- und ergebnisoffener Prozess“ zu verstehen ist. Im Zusammenhang mit Migrationsgründen, Migrationsgeschichten und Rechtstatus ist für Deutschland in den einschlägigen Diskursen die Unterscheidung zwischen Arbeitsmigranten, Aussiedlern und Flüchtlingen eingeführt, wobei diese Typisierung kritisch diskutiert werden kann (Treibel 2011). Der Integrationsbegriff ist in zweifacher Weise zu lesen: (a) Integration im Sinne von Zugängen von Migranten in die Funktionssysteme und Lebenszusammenhänge 
der Gesellschaft (,Sozialintegration '). Integration kann in dieser Lesart verstanden werden als die Eröffnung von gesamtgesellschaftlichen Teilhabechancen. (b) Davon zu unterscheiden ist die Integration der Gesellschaft. In dieser Lesart geht es um den sozialen und gesellschaftlichen Zusammenhalt vor dem Hintergrund wachsender Heterogenität ('Diversität') (,Systemintegration') (für einen Überblick der wissenschaftlichen Integrationsdebatte vgl. Filsinger 2014).

Im Hinblick auf unsere Fragestellung ist die rechtliche Dimension im Politikfeld der Migration und Integration von Bedeutung, wobei die Bundespolitik im föderalen System der Bundesrepublik Deutschland insbesondere im Bereich Zuwanderung, Aufenthalt und Asyl den gesetzlichen Rahmen bildet (Bommes 2018 [2009]; Schammann 2015). So regelt beispielsweise das Zuwanderungsgesetz Zuwanderung und Aufenthalt. Auch die neueren Asylgesetze („Asylpakete“) der letzten Jahre sind zu nennen, die nicht nur asylrechtliche Regelungen geändert haben, sondern auch die Aufgaben an Kommunen und Länder ausdifferenziert haben. So hat das Asylpaket I im Jahre 2015 u.a. den Ländern und Kommunen mehr Möglichkeiten im Bereich der Unterbringung eröffnet. Auch eine finanzielle Entlastung bzw. Unterstützung wurde in diese Regelung eingebaut (Bundesregierung 2015b). Das darauffolgende Asylpaket II verschärfte allerdings insbesondere Aufenthalts und Zuzugsbedingungen im Bereich des Familiennachzugs (Bundesregierung 2016).

Schammann (2015) weist allerdings zu Recht darauf hin, dass auch die Bundesländer aufenthaltsrechtliche Kompetenzen besitzen. Insbesondere die Diskussion um nicht vollzogene Abschiebungen von Seiten der Länder ist ein deutlicher Hinweis auf deren Kompetenzen im politischen Mehrebenensystem. Ferner spielen die Länder mit ihren Kompetenzen im Bereich der Bildungspolitik eine zentrale Rolle. Bildung ist als ein zentraler Bestandteil der Integration in Gesellschaft und Wirtschaft zu betrachten. Darüber hinaus 
erkennt Schammann (2015), dass sowohl im Bereich der Teilhabe als auch im Aufenthaltsrecht der Spielraum der Länder durchaus erheblich ist. Darüber hinaus werden kommunale Akteure immer wieder als wichtige Akteure hervorgehoben, was sich nicht zuletzt darauf zurückführen lässt, dass die unmittelbaren Folgen von Zuwanderung - ebenso wie der demografische Wandel - lokal bewältigt werden müssen (Filsinger 2018a). So können Kommunen insbesondere über die lokale Wirtschaftsförderung Akzente setzen. Ferner spielen sie bei der Bereitstellung öffentlicher Infrastruktur und der lokalen Daseinsfürsorge eine gewichtige Rolle (Grohs/Reiter 2014). Dennoch können kommunale Akteure nicht alle Parameter der Integration beeinflussen, da diese in die Migrations- und Integrationspolitiken der Europäischen Union, des Bundes und der Länder eingebunden sind (Bommes 2018 [2009]; Scholten/Penninx 2016). Gleichwohl führt die Tatsache, dass die Bundesländer bestimmte Aufgaben im Bereich Aufenthalt (Abschiebehemmnisse) und Integration an die Kommunen delegieren, zu Ermessensspielräumen. Diese werden in zunehmendem Ausmaß genutzt, um eigene Akzente zu setzen.

Systematisch lassen sich beispielsweise kommunale Integrationspolitiken in Deutschland seit den 1990er Jahren rekonstruieren, während die Initiativen seit den 1970er Jahren nicht als konzeptionelle oder strategische Entscheidungen von Seiten der Kommunalpolitik zu betrachten sind (Filsinger 2018a). Vielmehr handelte es sich hierbei überwiegend um Notlagenarbeit zur Bearbeitung der Folgen der Zuwanderung. Seit den frühen 2000er Jahren ist nun in vielen Städten und Landkreisen eine strategische Neuorientierung der kommunalen Integrationspolitik zu beobachten, die zunehmend auf eine interkulturelle Stadtpolitik setzt, die Vielfalt positiv würdigt und eine Willkommenskultur fördert. Hervorzuheben ist besonders die Implementation eines umfassenden strategisch und methodisch fundierten Integrationsmanagements, welches sich auf alle $\mathrm{Zu}$ - bzw. Eingewanderten bezieht, d.h. EUZuwanderer, Fachkräfte oder Geflüchtete (Filsinger 2017; 2018b Gesemann/Roth 2017). 
Dieser Wandel lässt sich nicht zuletzt auch auf eine Neuausrichtung der bundespolitischen Integrationspolitik zurückführen (Filsinger/Gesemann 2017). Darüber hinaus fördern Bundesund Landespolitik mit verschiedenen Programmen integrationspolitische Maßnahmen auf kommunaler Ebene (Filsinger 2018a).

\section{Zuwanderungsstrategien auf kommunaler Ebene?}

Im Zusammenhang mit Migration im Allgemeinen und (gezielter) Zuwanderung im Besonderen wird häufig der demografische Wandel genannt. Der empirische Befund, dass sich die Alters- und Bevölkerungsstruktur der Bundesrepublik in Zukunft stark verändern wird, ist in der politischen und gesellschaftlichen Debatte allgegenwärtig. Das Schlagwort des demografischen Wandels bezeichnet einen umfassenden sozialen Strukturwandel, der die gesamte Gesellschaft und ihre Teilsysteme betrifft und Antworten von den verschiedensten Akteuren erfordert (Bundesregierung 2015a). Eine Folge der Alterung und des Rückgangs der Bevölkerung ist das sinkende Erwerbspersonenpotential. Ein sinkendes Erwerbspersonenpotential stellt für die Wirtschaft und auch für die sozialen Sicherungssysteme eine Herausforderung dar (Bundesregierung 2015a). Eine prominente Gegenmaßnahme wird in weiterer qualifizierter Zuwanderung auf der Grundlage eines Einwanderungsgesetzes gesehen, welches insbesondere qualifizierte Fachkräfte anwerben und deren Integration vereinfachen soll (SVR 2018). Zunehmend wird auch in der Wissenschaft diskutiert, ob kommunale Akteure gezielte Anwerbungsversuche starten können, um der durch den demografischen Wandel hervorgerufenen Schrumpfung entgegenzuwirken (Kühn 2018). Dies gilt umso mehr, als dass lokale Akteure die Folgen des demografischen Wandels bearbeiten müssen. Die lokale Daseinsvorsorge und Infrastruktur hängt auch von der Bevölkerungsentwicklung ab. Folglich sollten Städte ein Interesse an (gezielter und geplanter) Zuwanderung haben, um die Folgen des Wandels abzumildern (Kühn 2018). Auf der anderen Seite zeigt sich, dass der demografische Wandel nicht alle Regionen und Städte in gleichem 
Maß und in gleicher Weise betrifft. Großstädte wie München, Berlin oder Frankfurt am Main weisen wachsende Einwohnerzahlen auf, während Regionen in Ost- aber auch Westdeutschland schrumpfen (Kühn 2017). Dementsprechend ist denkbar und plausibel, dass Kommunen ein Zuwanderungskonzept zur gezielten Anwerbung qualifizierter Menschen verabschieden, um somit Schrumpfungstendenzen und den damit verbundenen Auswirkungen zumindest entgegenzuwirken (Berding 2007; Kühn 2018). Insbesondere in Verbindung mit einem Einwanderungsgesetz auf Bundesebene, das den Ländern und Kommunen einen rechtlichen Rahmen für eine Anwerbung zur Verfügung stellen könnte, ist die strategische Ausrichtung einer „,kommunalen Zuwanderungspolitik“ in Form von Zuwanderungskonzepten grundsätzlich erwartbar. Allerdings sind die Chancen für die wirtschaftsstarken und prosperierenden Regionen um einiges besser als für die Regionen, die Zuwanderung stärker benötigen (BBSR 2017b; Bürk/Fischer 2013).

Im Kontext der demografischen Veränderungen und der häufig erhobenen Forderung nach Zuwanderung als Gegenmittel und der Annahme, dass Kommunen zur Bearbeitung der demografischen Probleme kommunale Zuwanderungskonzepte verabschieden, stellt sich nun die Frage, ob dies auch empirisch der Fall ist und Kommunen hier eine aktive Rolle spielen, indem sie beispielsweise Zuwanderungskonzepte entwerfen.

\section{Zuwanderungskonzepte in der BRD: Ergebnisse einer systematischen Recherche}

Die folgenden Erkenntnisse beruhen methodisch auf einer systematischen Internetrecherche zu kommunalen Zuwanderungskonzepten. Zusätzlich wurden vorhandene Konzepte einer ersten Dokumentenanalyse unterzogen. Gegenstand der Recherche waren alle Landkreise und kreisfreien Städte der Bundesrepublik Deutschland ${ }^{1}$. Als Suchbegriffe wurden zunächst „Zuwanderungskonzept“ und „Zuwanderungsstrategie“ in Kombination mit dem jeweiligen

\footnotetext{
${ }^{1}$ www.regionalstatistik.de
} 
Landkreis/der jeweiligen kreisfreien Stadt verwendet und anschließend auf den Internetseiten der Kommunen gesucht. Sofern diese keine Resultate lieferten, wurde lediglich „Zuwanderung“ bzw. „Migration“ auf den Internetseiten der Verwaltungen eingegeben und die Ergebnisse nach strategischen Konzepten durchsucht. Dabei wurde insbesondere darauf geachtet, dass explizit eine strategische Ausrichtung bzgl. weiterer Zuwanderung vorhanden war und sich dies in einem Konzept, Plan o.ä. niederschlug. Diese Vorgehensweise ist restriktiv und soll somit verhindern, dass verschiedene Maßnahmen in eine Analysekategorie gezwängt werden. Dies hat jedoch den Nachteil, dass wir uns lediglich auf

Zuwanderungskonzepte beschränken, jedoch eine Überdehnung dieser konzeptionellen Grundlagen verhindern. Insgesamt haben wir somit einen ersten Überblick über alle Landkreise und kreisfreien Städte und dortige Zuwanderungskonzepte.

Unsere Analyse aller Landkreise zeigt, dass explizite „Zuwanderungskonzepte“ in deutschen Kommunen kaum vorhanden sind. Insgesamt ergab sie, dass drei Landkreise ein „Zuwanderungskonzept“ verabschiedet haben. Dies gilt zumindest, wenn man nur solche Dokumente einbezieht, die mit unserer eng begrenzten Definition übereinstimmen. Bei genauerer Betrachtung der eruierten Konzepte fällt der Landkreis Potsdam-Mittelmark auf. Im September 2016 hat dieser Landkreis eine Zuwanderungsstrategie verabschiedet (Landkreis Potsdam-Mittelmark 2016). Den Hintergrund dieser Strategie bilden die Fluchtbewegungen der Jahre 2015 und 2016, die zu einem beträchtlichen Anstieg des Wanderungssaldos geführt haben. So ist eindeutig, dass dieses Konzept als Reaktion auf die externen, globalen Bedingungen zu sehen ist. Dies scheint die Einschätzung von Bommes (2018 [2009]) zu bestätigen, dass (lokale) Politik von globalen Entwicklungen abhängig ist. In dieser (quasi) funktionalistischen Logik liefert die lokale Politik lediglich reaktive Antworten auf die Veränderung in den sozioökonomischen Rahmenbedingungen. Das Konzept des Landkreises konzentriert sich zunächst eindeutig auf Integrationsleistungen und weniger auf die 
Anwerbung zukünftiger Zuwanderer. Dies lässt sich auch an den inhaltlichen Dimensionen ablesen: Wohnen, Arbeit, Sprachunterricht, (Aus-)Bildung und interkulturelle Öffnung. Deshalb bleibt offen, ob dieses Konzept tatsächlich der Anwerbung und Steuerung zukünftiger Zuwanderung dient oder vielmehr als strategische Ausrichtung der Integrationspolitik gesehen werden muss (siehe unten). Dieser Eindruck scheint sich zu verstärken, wenn man im Blick behält, dass die Fluchtbewegungen der Jahre 2015 und 2016 nicht „geplant“ waren. Vielmehr noch, weisen doch die Geflüchteten ein anderes Profil als beispielsweise angeworbene Fachkräfte auf (Brücker et al. 2016).

Auf der anderen Seite verlangt der Beschluss des Kreistages eindeutig eine „umsichtige und vorausschauende Migrations- und Integrationspolitik“, was auf eine erwünschte Steuerung künftiger Migration hindeutet (Landkreis Potsdam-Mittelmark 2016: 5). Dementsprechend verfolgt das Konzept zwei Ziele: Erstens eine intensive und strategische Bearbeitung bereits erfolgter Zuwanderung und zweitens eine strategische Ausrichtung und - sofern möglich eine Steuerung künftiger Migration. Inwiefern diese Steuerung im Zusammenhang mit dem demografischen Wandel steht, wird nicht explizit thematisiert.

Ein anderer Landkreis, der sich erklärtermaßen mit der Anwerbung von Zuwanderern befasst, ist der Landkreis Görlitz in Sachsen. Neben Themenfeldern wie (Aus-)Bildung und Familienfreundlichkeit geht es hier explizit auch um Anwerbung und die Etablierung einer Willkommenskultur. Das Konzept entstand im Rahmen der Fachkräfteallianz im Landkreis Görlitz und zeigt somit exemplarisch auf, dass nicht nur staatliche Akteure, sondern auch Wirtschaft und Gesellschaft in diesem Politikfeld eine gewichtige Rolle spielen. So weist das Konzept einerseits auf die Wichtigkeit eines Arbeitsplatzes für die Zugewanderten hin, aber andererseits auch auf die hohe Relevanz sozialinfrastruktureller und soziokultureller Bedingungen, um ein langfristiges Verbleiben in der Region zu sichern (Landkreis Görlitz 2016). 
In eine ähnliche Richtung geht das Landratsamt des Erzgebirgskreises (Landratsamt Erzgebirgskreis 2017). Zwar handelt es sich bei dessen Konzept um ein Integrationskonzept (siehe unten), jedoch zielt es eindeutig auch auf die Anwerbung weiterer Fachkräfte in die Region ab. Dies wird explizit mit den Folgen des demografischen Wandels begründet: „Vor dem Hintergrund der sinkenden Einwohnerzahl und des Fachkräftemangels müssen Zuwanderer spüren, dass sie in der Region gewollt und willkommen sind.“(Landratsamt Erzgebirgskreis 2017: 13). Hier zeigt sich der eindeutige Link zwischen demografischem Wandel und dem Wunsch nach künftiger Zuwanderung zur Bewältigung des Fachkräftemangels. Die Anwerbung wird hier als dreistufiger Prozess verstanden, der von der lokalen Politik, aber auch von den Akteuren der lokalen Wirtschaft und Zivilgesellschaft getragen wird. So beziehen sich diese Maßnahmen auf das Anwerben von Fachkräften. Anwerbung wird als Aufgabe der lokalen Wirtschaft gesehen, während das Welcome Center des Kreises für das Ankommen zuständig ist. Integration bildet die letzte Stufe des Prozesses und verweist auf das Halten der Zugewanderten in der Region (Einwanderung) durch die Eröffnung von Teilhabechancen an der lokalen Gesellschaft (Landratsamt Erzgebirgskreis 2017: 12). Dieses Konzept zeigt somit den Möglichkeitenraum einer kommunalen Zuwanderungssteuerung auf, in dem diese konzeptionell mit einer Integrationsstrategie verknüpft wird. Ferner werden verschiedene Akteure miteingebunden (Politik, Wirtschaft, Zivilgesellschaft), die mit unterschiedlichen Konzepten und Maßnahmen Migration und Integration zu steuern versuchen. Die Willkommenskultur ist als zentraler Bestandteil zu sehen, da diese sowohl ein möglicher Anziehungsfaktor für Zuwanderer ist, als auch der Integration und dem Verbleib im Landkreis dienen soll. So ist das Welcome Center des Kreises für die Koordination der Integrationsbemühungen von Unternehmen und Gesellschaft verantwortlich. Vorrangig ist folglich die Integration der Zugewanderten, die einen Verbleib in der Region impliziert (Landratsamt Erzgebirgskreis 2017: 14). 
Interessanterweise finden sich alle drei gefundenen Zuwanderungskonzepte in ostdeutschen Landkreisen. Dies spricht grundsätzlich für den starken demografischen Problemdruck, andererseits kann bei drei aufgefundenen Konzepten noch nicht von einem systematischen Zusammenhang gesprochen werden. Die explizite Verknüpfung und Forderung nach Zuwanderung scheint nach unseren Recherchen jedoch eher eine Ausnahme zu sein. Obgleich in der Forschung ein sog. local turn in der Migrationspolitik beobachtet wird (Hackett 2017), lässt sich dieser bisher empirisch in Bezug auf die strategische Anwerbung und somit Steuerung von zukünftiger Zuwanderung in Deutschland nur vereinzelt feststellen. Dieser Befund ergibt sich, wenn man explizit nach Zuwanderungsstrategien sucht. Nicht ausgeschlossen werden kann, dass einige Landkreise und Städte Zuwanderungspolitiken in (Stadt- bzw. regionale-) Entwicklungskonzepte integrieren. Beispielhaft kann hier das integrierte ländliche Entwicklungskonzept des Landkreises Vorpommern-Greifswald genannt werden, welches den „Erhalt und [das] Anwerben von Fachkräften“ als explizites Ziel der Kreisentwicklung festhält (Landkreis Vorpommern-Greifswald 2016: 180). Nach den Ergebnissen unserer Recherche scheint es jedoch auch hier nicht überall eine Integration von Zuwanderungskonzepten in die Kreisentwicklung zu geben.

Es ist davon auszugehen, dass die gewählten Suchbegriffe „Zuwanderung“ und „Migration“ zumindest in Teilen solche Kapitel auf den Websites der Verwaltung auffinden lassen würden. Deshalb erscheint eine weitere Forschung, die Querschnittskonzepte genauer untersucht, geeignet, unsere vorläufigen Ergebnisse zu erweitern.

\section{Steuerung durch Integration?}

Die Recherche zu Integrationskonzepten erfolgte nach dem gleichen Muster wie die Recherche zu den Zuwanderungskonzepten. Genutzt wurden die Begriffe „Integrationskonzept“ und „Integration“ (siehe auch Filsinger/Gesemann 2017). Integrationskonzepte dienen insbesondere der Systematisierung der Integrationsbemühungen 
auf kommunaler Ebene. So können anhand des Konzeptes Ziele festgeschrieben und ihre Erreichung in Form von Monitoring und Evaluationen überprüft werden (Filsinger/Gesemann 2017; Filsinger 2018a).

Betrachtet man die Ergebnisse der Internetrecherche zu Integrationskonzepten, so zeigt sich, dass 177 Landkreise und kreisfreie Städte (circa 44 Prozent) über ein Integrationskonzept verfügen. Kommunale Integrationskonzepte spielen eine immer größere Rolle und dies nicht nur in Groß- und Vorreiterstädten (Filsinger/Gesemann 2017). Regressionsanalysen zeigen, dass Integrationskonzepte vor allem in Großstädten und dichter besiedelten Landkreisen bzw. urbanen Ballungsräumen vorhanden sind. Ein Unterschied zwischen West- und Ostdeutschland ließ sich nicht feststellen. Der Ausländeranteil spielt dem Anschein nach keine Rolle. Es zeigt sich in der Literatur, dass die kommunale Ebene im Bereich der Integrationspolitik nicht selten eigene Akzente setzt, die in manchen Fällen nationalstaatlichen Regelungen entgegenlaufen können (Jørgensen 2012; Schmidtke/Zaslove 2014; Dekker et al. 2015). Insgesamt lässt sich im letzten Jahrzehnt ein Schub in der Entwicklung, Profilierung und Implementation integrierter, kommunalpolitisch legitimierter Gesamtkonzepte mit interkultureller Orientierung beobachten. Diese implizieren deren administrative Institutionalisierung in von Kommune zu Kommune unterschiedlichen Formaten sowie die Umsetzung eines (sozialräumlichen) und wirkungsorientierten Integrationsmanagements (Gesemann/Roth/Aumüller 2012; Filsinger/Gesemann 2017; Filsinger 2018a; 2018b, Reichwein 2018; SVR 2018 „Mit unterschiedlichen Geschwindigkeiten und Akzentsetzungen sind die Kommunen in der Etablierung einer ,Willkommenskultur' engagiert, die auf dem Konzept der interkulturellen Öffnung der Verwaltungen und der lokalen Institutionen basiert, in Kenntnisnahme und Erwartung weiterer Zuwanderung aus der EU und der Fluchtzuwanderung“ (Filsinger/Gesemann 2017: 27; siehe auch SVR 2012; 2018). 
Dass diese Konzepte in der Erwartung weiterer Zuwanderung erarbeitet werden, darauf deutet nicht zuletzt auch die mittlerweile verbreitete Evaluation und Fortschreibung der Konzepte hin (Filsinger/Gesemann 2017). So hat die Stadt Flensburg ihr Integrationskonzept von 2010 mit drei Fortschreibungen fortentwickelt und angepasst, um auf neue Entwicklungen und veränderte Aufgabenstellungen eingehen zu können (Stadt Flensburg 2010). Darüber hinaus wurde 2016 ein Konzept für die Jahre 2016 bis 2022 verabschiedet, welches sich kontinuierlich weiterentwickeln soll (Stadt Flensburg 2016). Hieran lässt sich die Flexibilität kommunaler Konzepte zeigen, die einerseits klare Handlungsempfehlungen geben, sich aber andererseits bei Bedarf auch problemorientiert überarbeiten, anpassen und weiterentwickeln lassen.

Nicht zu vernachlässigen sind vielfältige Formen der Förderung der Partizipation von Migrantinnen und Migranten in der Stadtpolitik, die Aktivierung und Unterstützung zivilgesellschaftlichen Engagements sowie Maßnahmen zur Antidiskriminierung. In den Policy-Prinzipien ist ein Perspektivwechsel von einem Defizit- zu einem Kompetenz- bzw. Potenzialansatz zu beobachten (Filsinger 2018a). Betont werden die Ressourcen von Migrantinnen und Migranten. Zuwanderung wird zunehmend als Chance für die Stadtentwicklung begriffen. In diesem Zusammenhang ist auf die Weiterentwicklung kommunaler Integrationspolitiken in einer beachtlichen Zahl von Städten zu interkulturellen Stadtpolitiken bzw. zu Diversitätspolitiken hinzuweisen (Filsinger 2018a; 2018b). Einzelne Studien weisen gleichwohl auf zum Teil deutliche Widerstände, interkulturelle Konflikte und Ambivalenzen im Umgang mit Zuwanderung bzw. auf die anspruchsvollen Voraussetzungen für solche Politiken hin (Gestring 2014; Krummacher 2017; Schulze-Böing 2017).

Um neue Zuwanderung zu gewinnen bedarf es nämlich guter Ankunfts- und Aufnahmesowie attraktiver Lebensbedingungen (Berding 2007). Selbstverständlich betrifft dies zunächst einmal ökonomische Faktoren wie Arbeitsplätze, aber auch Wohnraum oder die 
öffentliche Infrastruktur im Bildungs- und Sozialbereich (Filsinger, M 2017; Jayet et al. 2016). Diese Faktoren sind selbstverständlich nur zu einem bestimmten Grad von der Kommunalpolitik beeinflussbar. Die lokale Wirtschaftsförderung und Bildungs- und Sozialprogramme sowie die Wohnungsversorgung und Sozialraumgestaltung sind gleichwohl Beispiele wie hier steuernd eingegriffen werden kann und somit die Attraktivität für bereits angekommene sowie potentielle Zuwanderer gesteigert werden kann (Bommes 2018 [2009]).

\section{Zusammenfassung}

Mit unserer Internetrecherche gingen wir der Frage nach, ob kreisfreie Städte und Landkreise in der Bundesrepublik Deutschland ihren Handlungsspielraum nutzen, um Zuwanderungskonzepte zu entwickeln, die aktiv qualifizierte Zuwanderung fördern, um den Folgen des demografischen Wandels entgegenzuwirken. Vor dem Hintergrund des Problemdrucks in vielen Städten und Regionen sowie der in der wissenschaftlichen wie in der öffentlichen Diskussion herrschenden Übereinstimmung hinsichtlich der Notwendigkeit weiterer Zuwanderung wäre die Nutzung von Zuwanderungskonzepten auf kommunaler Ebene zu erwarten (Kühn 2018). Städte und Landkreise haben zwar nur eingeschränkte Spielräume für eine aktive Zuwanderungspolitik, da sie von staatlichen Migrationsregimen in Bund, Ländern und EU abhängig sind (Grohs/Reiter 2014; Scholten/Penninx 2016; Thränhardt 2018), aber wie die Empirie zeigt sind solche Handlungsspielräume vorhanden (Filsinger/Gesemann 2017).

Unsere Internetrecherche aller Landkreise und kreisfreien Städte zeigt, dass lediglich drei Landkreise in Deutschland zu ermitteln waren, die ein Konzept verabschiedet haben, dass unseren Kriterien entspricht. Eine aktive und strategisch ausgerichtete Zuwanderungsförderung mit Hilfe von Konzepten ist dementsprechend auf der kommunalen Ebene weitgehend nicht gegeben. Wir führen die geringe Verbreitung hauptsächlich auf zwei Faktoren zurück. Erstens, bildet die Widersprüchlichkeit der Migrationspolitik auf 
Bundesebene einen unsicheren Rahmen für kommunalen Entscheidungen und befördert so eine zurückhaltende Anwerbepolitik. In Anschlag zu bringen ist auch die gestiegene Skepsis gegenüber weiterer Zuwanderung in beachtlichen Teilen der Bevölkerung. Andererseits, sind viele Ressourcen auf der kommunalen Ebene im Feld der Integrationspolitik und Integrationsarbeit gebunden, die sich mit den liegen gebliebenen Aufgaben im Zusammenhang mit früheren Zuwanderungen bzw. erfolgter Einwanderungen sowie mit den Folgen der jüngsten Zuwanderungsbewegungen (Fluchtzuwanderung, EU-(Armuts)Migration) auseinanderzusetzen haben.

Unsere erweiterte Recherche zeigt auf, dass strategische Integrationskonzepte weitaus stärker verbreitet sind. So haben circa 44 Prozent der kreisfreien Städte und Landkreise ein Integrationskonzept verabschiedet. Diese strategische Ausrichtung der Integrationspolitik ist insbesondere in Großstädten und dichter besiedelten Landkreisen zu finden. So scheint der Fokus der kommunalen Akteure insbesondere auf der Integration bereits Zugewanderter zu liegen und weniger auf der Anwerbung. Betrachtet man die Inhalte der Konzepte so ist zu erkennen, dass deren Fokus auf die klassischen Felder der Sozialintegration gerichtet ist: Sprache, Bildung, Arbeit, Wohnen und soziale Teilhabe. Darüber hinaus spielen die interkulturelle Öffnung und die Etablierung einer Willkommenskultur für Ankommende eine besondere Rolle. Die neueren bzw. weiterentwickelten Integrationskonzepte sind aber häufig so ausgerichtet, dass sie Steuerungspotenziale gewonnen haben. Insofern sind viele Kommunen auf erwartbare weitere Zuwanderung strategisch vorbereitet. Die - zumindest in Vorreiterstädten und -Landkreisen - beobachteten Integrations- und Diversitätspolitiken lassen auch Ansätze für eine Zuwanderungsstrategie erkennen (Filsinger/Gesemann 2017).

\section{Literaturverzeichnis}


Berding, U (2007). Migration - ein Thema für die Stadtentwicklungspolitik. Dissertation vorgelegt an der Rheinisch-Westfälischen Technischen Hochschule Aachen.

Bommes, M (2018) [2009]. Die Rolle der Kommunen in der bundesdeutschen Migrationsund Integrationspolitik. in: Gesemann, F; Roth, R (Hrsg.): Lokale Integrationspolitik in der Einwanderungsgesellschaft, Migration und Integration als Herausforderung von Kommunen. 2. Auflage. Wiesbaden: VS-Verlag für Sozialwissenschaften, S. 99-123.

Brücker, H; Fendel T; Kunert, A; Mangold, U; Siegert, M; Schupp, J (2016): Warum sie kommen, was sie mitbringen und welche Erfahrungen sie machen. In: IAB Kurzbericht: Aktuelle Analysen aus dem Institut für Arbeitsmarkt- und Berufsforschung, 15/2016.

Bundesamt für Migration und Flüchtlinge (2017): Asylgeschäftsbericht für den Monat Juli 2017. Bundesamt für Migration und Flüchtlinge.

Bundesinstitut für Bau-, Stadt-, und Raumforschung (BBSR) (2017): Raumordnungsbericht 2017. Daseinsvorsorge sichern. Sonderveröffentlichung Bonn, Oktober 2017.

Bundesinstitut für Bau-, Stadt- und Raumforschung (BBSR) (2017b): Regionale Implikationen der Zuwanderung aus dem Ausland in Deutschland. Bonn: BBSR-OnlinePublikation Nr. 044/2017.

Bundesregierung (2015a): Die Demografiestrategie der Bundesregierung. https://www.bundesregierung.de/breg-de/service/newsletter-und-abos/fakten-zurregierungspolitik/die-demografiestrategie-der-bundesregierung249788?view=renderNewsletterHtml.

Bundesregierung (2015b): Gesetzespaket in Kraft getreten. Effektive Verfahren, frühe Integration. https://www.bundesregierung.de/Content/DE/Artikel/2015/10/2015-10-15-asylfluechtlingspolitik.html. 
Bunderegierung (2016): Asylpaket II in Kraft. Kürzere Verfahren, weniger Familiennachzug. https://www.bundesregierung.de/Content/DE/Artikel/2016/02/2016-02-03-asylpaket2.html.

Bürk, T; Fischer, S (2013): Zuwanderung aus dem Ausland - Eine Perspektive für Städte des peripherisierten Raums? in: Bernt, M; Liebmann, H (Hrsg.): Peripherisierung, Stigmatisierung, Abhängigkeit? Deutsche Mittelstädte und ihr Umgang mit Peripherisierungsprozessen. Wiesbaden: Springer-VS, S. 178-192.

Dekker, R; Emilsson, H; Krieger, B; Scholten, P (2015): A Local Dimension of Integration Policies? A Comparative Study of Berlin, Malmö, and Rotterdam. International Migration Review 49(3): S. 633-658.

Filsinger, D (2014): Integration - ein Paradigma ohne Alternative? In: Alisch, M (Hrsg.): Älter werden im Quartier: Soziale Nachhaltigkeit durch Selbstorganisation und Teilhabe. Kassel: university press, S. 169-196

Filsinger, D (2017a): Soziale Arbeit mit Flüchtlingen. Strukturen, Konzepte und Perspektiven. WISO Diskurs. Bonn: Friedrich-Ebert-Stiftung.

Filsinger, D (2017b): Interkulturelle Öffnung der Kommunen. in: Scherr, A; El-Mafaalani, A; Gökcen, E (Hrsg.): Handbuch Diskriminierung. Wiesbaden: Springer VS, S. 639-656.

Filsinger, D (2018a): Entwicklung, Konzepte und Strategien der kommunalen Integrationspolitik, in: Gesemann, F; Roth, R (Hrsg.): Handbuch Lokale Integrationspolitik. Wiesbaden: VS-Verlag für Sozialwissenschaften, S. 279 - 296.

Filsinger, D (2018b): Kommunale Integrationspolitiken: Entwicklungsmuster, Bilanzierung und Perspektiven. In: Migration und Soziale Arbeit, 40(3), S. 196-203. 
Filsinger, D; Gesemann, F (2017): Kommunale Integrationsleitbilder: Verbreitung, Funktion und Wirksamkeit. Expertise im Auftrag des SVR, Berlin/Saarbrücken.

Filsinger, M (2017): Deutschland ein Einwanderungsland, aber nicht überall? -

Determinanten der geographischen Verteilung von Migrantinnen und Migranten. In: Henn, D; Prigge, J; Ries, K; Lück-Filsinger, M (Hrsg.) (2017): Streifzüge durch die angewandte Sozialwissenschaft: Evaluation - Migration - Sozialpolitik - Soziale Arbeit Dieter Filsinger zum 65. Geburtstag. Münster: Waxmann-Verlag, S. 171 - 186.

Gesemann, F; Roth, R (2017): Erfolgsfaktoren der kommunalen Integration von Geflüchteten. Berlin: Friedrich-Ebert-Stiftung.

Gesemann, F; Roth, R; Aumüller, J (2012): Stand der kommunalen Integrationspolitik in Deutschland. Berlin: Bundesministerium für Verkehr, Bau und Stadtentwicklung und Beauftragte der Bundesregierung für Migration, Flüchtlinge und Integration.

Gestring, N (2014): Widersprüche und Ambivalenzen kommunaler Integrationskonzepte. In: Gans, Paul (Hrsg.): Räumliche Auswirkungen der internationalen Migration. Hannover: Akademie für Raumforschung und Landesplanung, S. 311-326.

Grohs, S; Reiter, R (2014): Kommunale Sozialpolitik. Handlungsoptionen bei engen Spielräumen. WISO Diskurs. Bonn: Friedrich-Ebert-Stiftung.

Hackett, S E (2017): The „local turn” in historical perspective: two city case studies in Britain and Germany. International Review of Administrative Sciences, 83 (29), S. 340-357.

Jayet, H; Rayp, G; Ruyssen, I; Ukrayinchuck, N (2016): Immigrants`location choice in Belgium. The Annals of Regional Science, 57(1), S. 63-89. 
Jørgensen, M B (2012): The Diverging Logics of Integration Policy Making at National and City Level. International Migration Review 46(1): S. 244-278.

Krummacher, M (2017): Kommunale Integrationspolitik in Deutschland. Fakten, Entwicklungstrends, Widersprüche, Perspektiven. In: Geisen, T; Riegel, C; Yildiz, E (Hrsg.): Migration, Stadt und Urbanität. Perspektiven auf die Heterogenität migrantischer Lebenswelten. Wiesbaden: Springer-VS, S. 79-96.

Kühn, M (2017): Zuwanderung - eine Perspektive für schrumpfende Städte und Regionen?.

In Geteilte Räume. Strategien für mehr sozialen und räumlichen Zusammenhalt: Bericht der Fachkommission "Räumliche Ungleichheit" der Heinrich-Böll-Stiftung. Berlin: HeinrichBöll-Stifung, S. 58-78.

Kühn, M (2018): Immigration Strategies of Cities: Local Growth Policies and Urban Planning in Germany. European Planning Studies, 26(9), S. 1747-1762.

Kühn, M; Münch S (unveröffentlichtes Manuskript): Zuwanderungspolitik - ein neues kommunales Aufgabenfeld?

Landeshauptstadt Stuttgart (2018): Abteilung Integrationspolitik. https://www.stuttgart.de/img/mdb/item/645786/135657.pdf

Landkreis Görlitz (2016): Handlungskonzept der regionalen Fachkräfteallianz des Landkreises Görlitz.

Landkreis Potsdam-Mittelmark (2016): Zuwanderungsstrategie. Belzig.

Landkreis Vorpommern-Greifswald (2016): Integriertes Ländliches Entwicklungskonzept für den Landkreis Vorpommern-Greifswald. 
Landratsamt Erzgebirgskreis (2017): Integrationskonzept des Erzgebirgskreises. AnnabergBuchholz

Pries, L (2008): Migration. In: Farzin, S (Hrsg.): Lexikon Soziologie und Sozialtheorie. Hundert Grundbegriffe. Stuttgart: Reclam, S. 186-188

Pries, L (2013): Internationale Migration. Bielefeld: Transkript.

Putnam, R D (2007): E Pluribus Unum: Diversity and Community in the Twenty-first Century. The 2006 Johan Skytte Prize Lecture. Scandinavian Political Studies 30 (2), S. 137-174.

Reichwein, Alfred (2018): Kommunales Integrationsmanagement. Ansätze für eine strategische Steuerung der Integrationsarbeit. Gesemann, F; Roth, R (Hrsg.): Handbuch Lokale Integrationspolitik. Wiesbaden: VS-Verlag für Sozialwissenschaften, S. 693-704.

Sachverständigenrat deutscher Stiftungen für Integration und Migration (2012): Integration im föderalen System: Bund, Länder und Kommunen in Deutschland. Die Messung von Integration in Deutschland und Europa. Möglichkeiten und Grenzen bestehender Integrationsmonitorings. SVR-Bericht 2012-1. Berlin.

Sachverständigen Rat deutscher Stiftungen für Integration und Migration (2018): Steuern, was zu steuern ist: Was können Einwanderungs- und Integrationsgesetze leisten. Jahresgutachten 2018. Berlin.

Schammann, H (2015): Rette sich, wer kann? Flüchtlingspolitik im Föderalismus. Aus Politik und Zeitgeschichte, 25, S.26-31.

Schmidtke, O; Zaslove, A (2014): Why Regions Matter in Immigrant Integration Policies: North Rhine-Westphalia and Emilia-Romagna in Comparative Perspective. Journal of Ethnic and Migration Studies 40(12), S. 1854-1874. 
Scholten, P; Penninx, R (2016): The Multilevel Governance of Migration and Integration. In Garcés-Mascarenas, B; Penninx, R (eds.), Integration Processes and Policies in Europe, IMISCOE Research Series, Dordrecht: Springer, S. 91-108.

Schulze-Böing, Matthias (2017): Kommunale Integrationspolitik und Quartiersentwicklung: Möglichkeiten, Grenzen, Praxis. In: Bundesinstitut für Bau-, Stadt- und Raumforschung (Hrsg.): Regionale Implikationen der Zuwanderung aus dem Ausland in Deutschland. Bonn: BBSR-Online-Publikation Nr. 044/2017.

Stadt Flensburg (2010): Integrationskonzept für Menschen mit Migrationshintergrund mit Handlungsleitfaden 2010 - 2015.

Stadt Flensburg (2016): Zusammenleben in Flensburg - Integrationskonzept für Menschen mit Migrationshintergrund $2016-2022$.

Thränhardt, D (2018): Integrationspolitik in Deutschland zwischen Markt und Plan: Bund, Länder und Gemeinden. In: Gesemann, F; Roth, R (Hrsg.): Handbuch Lokale Integrationspolitik. Wiesbaden: VS-Verlag für Sozialwissenschaften, S. 823-834.

Treibel, A (2011): Migration in modernen Gesellschaften: Soziale Folgen von Einwanderung, Gastarbeit und Flucht. München und Weinheim: Juventa. 